\title{
Diffusion and advection model for particle transport in young pulsar wind nebulae
}

\author{
Xiaping Tang and Roger A. Chevalier \\ Department of Astronomy, University of Virginia, P.O. Box 400325, \\ Charlottesville, VA 22904-4325; \\ email: xt5ur@virginia.edu, rac5x@virginia.edu
}

\begin{abstract}
The magnetohydrodynamic (MHD) model for young pulsar wind nebulae (PWN) has been successful in reproducing many features of the nebulae. The model is characterized by a termination shock (TS) between the PWN and unshocked pulsar wind. Relativistic particles are injected at the TS and follow an advective flow to the outer boundary. However, toroidal structure of well studied young PWN like the Crab Nebula, 3C 58 and G21.5-0.9 is only present in the region close to the TS. In the outer parts of the nebulae, filamentary and loop-like structure is observed. Also, the radial variation of spectral index due to synchrotron losses is smoother than expected in the MHD flow model. We find that a pure diffusion model with energy independent diffusion and a transmitting boundary can reproduce the basic data on nebular size and spectral index variation for the Crab, 3C 58, and G21.5-0.9. Energy dependent diffusion is also discussed. Power law variations of the coefficient with energy are degenerate with variation in the input particle energy distribution index in the steady state case. Monte Carlo simulations of particle transport with both diffusion and advection for the Crab nebula and $3 \mathrm{C}$ 58 suggest a picture in which advection dominates the inner part of the PWN where toroidal structure is clearly present. Diffusion dominates the outer part of the PWN where filamentary and loop-like structure is observed. The source of the chaotic field is uncertain, but may be related to Rayleigh-Taylor instability at the outer boundary of young nebulae and/or the kink instability of the toroidal magnetic field.
\end{abstract}

Keywords. ISM: individual (Crab Nebula) —ISM: supernova remnants- pulsars: general stars: winds, outflows

\section{Introduction}

Pulsar wind nebulae (PWN), the result of interaction between relativistic pulsar winds and supernova $(\mathrm{SN})$ ejecta, are excellent sites for studying not only pulsar wind dynamics and shock processes but also particle transport and magnetic field evolution (Gaensler \& Slane 2006). The current standard picture for particle transport in young PWN is based on the magnetohydrodynamic (MHD) model first presented by Rees \& Gunn (1974) and then discussed in detail by Kennel \& Coroniti (1984a,b). In the MHD model, particles are accelerated at the termination shock and then follow the downstream advective flow toward the outer boundary. During this process, particles lose energy to synchrotron radiation and adiabatic expansion. The 1-dimensional MHD model by Kennel \& Coroniti (1984a,b) successfully explains the diminishing size of the Crab Nebula as the frequency increases and its integrated spectrum from infrared to X-ray wavelengths. The model also predicts a position of the termination shock that is consistent with X-ray observations (Kargaltsev \& Pavlov 2008). However, the jet torus structure observed in X-rays cannot be addressed in a 1D model, so attention has turned to 2D and even 3D MHD simulations of young PWN. In 2D simulations, the pulsar power is assumed to depend on pulsar angle; the simulations can reproduce the toroidal structure surrounding the central pulsar 
(Komissarov \& Lyubarsky 2003; Del Zanna et al. 2004) and time variability of the inner structure (Volpi et al. 2008; Camus et al. 2009; Komissarov \& Lyutikov 2011). The MHD model is successful in explaining many aspects of Crab Nebula and other young PWN, but recent observation of 3C 58 and G21.5-0.9 (Slane et al. 2000, 2008; Safi-Harb et al. 2001) show some features contradicting the MHD model. Both show toroidal structure very close to the central pulsar, but the radial variation of spectral index is smoother than expected in the standard picture. In Tang \& Chevalier (2012), we found that adding diffusion to the current picture of particle transport can explain many of these aspects. We propose that both advection and diffusion are important for particle transport in young PWN. Advection dominates particle transport in the region close to the central pulsar, while diffusion dominates the outer part of the PWN.

\section{Theoretical model}

Our work in Tang \& Chevalier (2012) is motivated by the Chandra observations of 3C 58 and G21.5-0.9 (Slane et al. 2000, 2008; Safi-Harb et al. 2001). The radial variation of spectral index is inconsistent with the MHD model but consistent with the profile from a diffusion model (Wilson 1972). We start with a pure diffusion model by Gratton (1972) for particle transport in young PWN with spherical symmetry, transmitting boundary and energy independent diffusion coefficient. We also assume that the magnetic field is constant within the PWN and synchrotron losses are the only energy loss. We use Gratton's model to fit the spectral index distribution of not only 3C 58 and G21.5-0.9 in $\mathrm{X}$-rays but also the Crab Nebula from radio to optical. We obtained a reduced $\chi^{2}$ about 0.8 for $3 \mathrm{C} 58$ and 3.3 for G21.5-0.9.

In Gratton's model, the nebular size of a PWN is determined by a critical frequency $\nu_{R} \propto D^{2} / R^{4} B^{3}$ where $D$ is the diffusion coefficient, $R$ is the radius of nebular outer boundary and $B$ is the magnetic field. When $\nu<\nu_{R}$, the nebular size remains the same due to the outer boundary condition. When $\nu>\nu_{R}$, the size tends to shrink as the cooling time of particles is smaller than the diffusion time. $B$ and $R$ are determined from observation and the diffusion coefficient $D$ can be obtained through model fitting. In our best fit models, $\nu_{R}=2 \times 10^{13} \mathrm{~Hz}$ for the Crab Nebula, $\nu_{R}=1.3 \times 10^{18} \mathrm{~Hz}$ for 3C 58, and $\nu_{R}=2.6 \times 10^{17} \mathrm{~Hz}$ for G21.5-0.9. For the Crab Nebula, X-ray, optical and near-IR frequencies are all in the $\nu>\nu_{R}$ regime, so the nebular size of the Crab decreases from radio to X-rays. For 3C 58 and G21.5-0.9, all frequencies below soft X-rays are in the $\nu<\nu_{R}$ regime, so the radio, optical and soft X-ray nebular sizes of 3C 58 and G21.5-0.9 tend to be similar.

We then calculated the size of the Crab Nebula as a function of frequency. The calculated nebular size is smaller than observed at high frequency. A possible reason is that Gratton's model doesn't take possible energy dependent diffusion into account. Our best fit diffusion coefficient for 3C 58 and G21.5-0.9 in X-rays is larger than the best fit coefficient of the Crab Nebula at optical wavelengths by at least one order of magnitude. If there is energy dependent diffusion, it could help explain the discrepancy in diffusion coefficient among them. Another reason is that in the high frequency band the nebular size is smaller, so advection is more important. Observations show that the toroidal structure is in the region close to central pulsar while filamentary structure dominates the outer part of nebula. We end up with a picture in which advection dominates particle transport in the region close to central pulsar while diffusion dominates the outer part of the PWN. In considering an energy dependent pure diffusion model, we found that power law variations of the diffusion coefficient with energy are degenerate with variation in the input particle energy distribution index in the steady state, transmitting boundary case. 


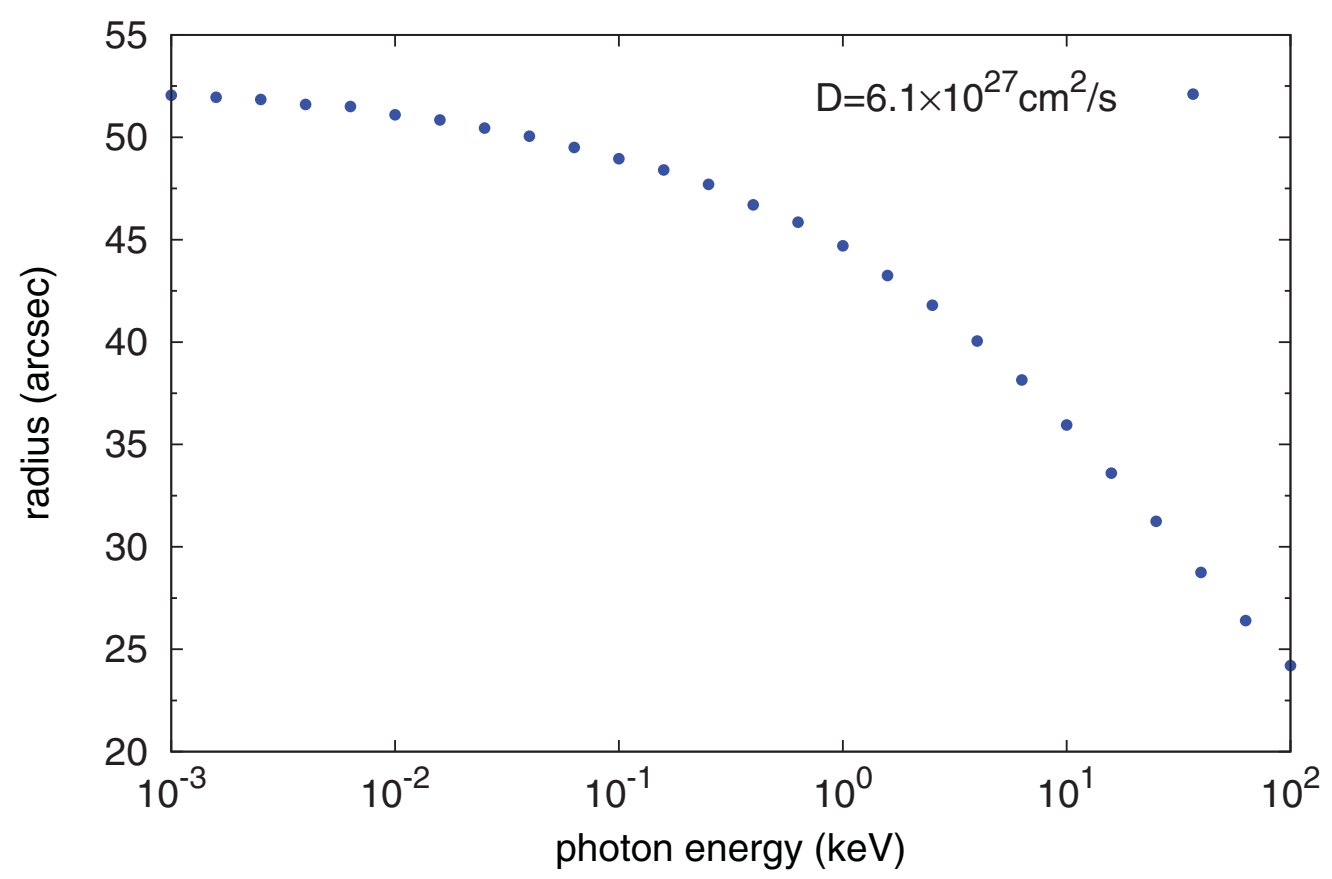

Figure 1. 3C 58 half-light radius based on a pure diffusion model with $B=80 \mu \mathrm{G}$ and $\mathrm{p}=2.93$ as in Tang \& Chevalier (2012).

Finally we carried out Monte Carlo simulations for both the Crab Nebula and 3C 58 with diffusion plus advection. In the Monte Carlo simulations, we took the finite size of the termination shock into account and tested the effect of different outer boundary conditions. The diffusion and advection model with a reflecting outer boundary gave the best fit for the spectral index distribution. Adding advection also helped fitting the nebular size behavior for the Crab Nebula.

In Tang \& Chevalier (2012), we mainly focused on fitting the spectral index distribution with different models, and gave only a brief discussion of the nebular size behavior of the Crab. Here we specifically consider the nebular size behavior of 3C 58. Based on Gratton's model, $\nu_{R}=1.3 \times 10^{18}$ for $3 \mathrm{C} 58$, which indicates that nebular size of $3 \mathrm{C} 58$ shrinks in hard X-rays. This effect may be detectable with the recently launched hard X-ray space telescope NuSTAR. Since 3C 58 has a smaller magnetic field and larger diffusion coefficient than the Crab Nebula, advection should be less prominent in $3 \mathrm{C} 58$ and pure diffusion may be a good approximation. We carried out calculations for the nebular size of 3C 58 as a function of photon energy based on a pure diffusion model (Figure 1). In the calculation we assume 3C 58 is associated with SN 1181. Our simulation results do not fit the observations well. The Chandra data show that the nebular size of 3C 58 does not shrink significantly around a few keV. We also carried out Monte Carlo simulations for 3C 58 with the best fit parameters from Tang \& Chevalier (2012). The results are shown in Table 1 . Monte Carlo simulation results are more consistent with the Chandra observations and can be used for future comparison with observations. Energy dependent diffusion, which will result in slower shrinking of nebular size as function of photon energy, is not included in the Monte Carlo simulation. 
Table 1. Half light radius of 3C 58 based on diffusion and advection model

\begin{tabular}{l|lllllll|}
\hline photon energy $(\mathrm{keV})$ & 1 & 1 & $\mid$ & 5 & 10 & 50 \\
\hline half light radius (arcsec) & $\mid$ & 55 & $\mid$ & 48 & $\mid$ & 43 & 31 \\
\hline
\end{tabular}

\section{Discussion}

Both diffusion and advection play an important role in particle transport in young PWN. Advection dominates the region close to central pulsar where toroidal structure is clearly observed. Diffusion dominates the outer part of PWN where filamentary structures are detected. A pure diffusion model tends to give a good fit for spectral index distribution and nebular size of young PWN when cooling is not important. When particles suffer strong synchrotron cooling and nebula size begin to shrink quickly, advection plays a greater role as the emission is dominated by the central region. The source of the chaotic field is uncertain, but may be related to the Rayleigh-Taylor instability at the outer boundary and/or the kink instability of the toroidal magnetic field.

\section{References}

Camus, N. F., Komissarov, S. S., Bucciantini, N., \& Hughes, P. A. 2009, MNRAS, 400, 1241

Del Zanna, L., Amato, E., \& Bucciantini, N. 2004, A\&GA, 421, 1063

Del Zanna, L., Volpi, D., Amato, E., \& Bucciantini, N. 2006, A\&A, 453, 621

Gaensler, B. M. \& Slane, P. O. 2006, ARAA, 44, 17

Gratton, L. 1972, Ap\&SSS, 16, 81

Kargaltsev, O. \& Pavlov, G. G. 2008, in 40 Years of Pulsars: Millisecond Pulsars, Magnetars and More, ed. C. G. Bassa, Z. Wang, A. Cumming, V. M. Kaspi (Melville, NY: AIP), 171

Kennel, C. F. \& Coroniti, F. V. 1984a, ApJ, 283, 694

Kennel, C. F. \& Coroniti, F. V. 1984b, ApJ, 283, 710

Komissarov, S. S. \& Lyubarsky, Y. E. 2003, MNRAS, 344, L93

Komissarov, S. S. \& Lyutikov, M. 2011, MNRAS, 414, 2017

Rees, M. J. \& Gunn, J. E. 1974, MNRAS, 167, 1

Safi-Harb, S., Harrus, I. M., Petre, R., Pavlov, G. G., Koptsevich, A. B., \& Sanwal, D. 2001, ApJ, 561, 308

Slane, P., Chen, Y., Schulz, N. S., Seward, F. D., Hughes, J. P., \& Gaensler, B. M. 2000, ApJ, 533, L29

Slane, P., Helfand, D. J., Reynolds, S. P., Gaensler, B. M., Lemiere, A., \& Wang, Z. 2008, ApJ, 676, L33

Tang, X. \& Chevalier, R. A. 2012, ApJ, 752, 83

Volpi, D., Del Zanna, L., Amato, E., \& Bucciantini, N. 2008, A\&AA, 485, 337

Wilson, A. S. 1972, MNRAS, 160, 355 